\title{
Research Algorithm on Edge Extraction of Images Using Cellular neural network method
}

\author{
Haiming Jing \\ School of Information Science and Technology \\ Shijiazhuang Tiedao University \\ Shijiazhuang, China \\ jimperlov@yeah.net
}

\author{
Ning Zhao \\ School of Economic and Management \\ Shijiazhuang Tiedao University \\ Shijiazhuang, China \\ zz_ning860@sohu.com
}

\begin{abstract}
Image edge extraction is a very important foundation in image analysis fields, such as image segmentation, target region recognition and region shape extraction, and plays an important role in engineering applications. The theory and analysis of image edge extraction with Cell Neural Network is in detailed described by its definition and theorem. By digital image processing algorithms, the right templates is found. then the binary image design flow chart of the algorithm is used to test the binary image edge. The detection results show these parameters are suitable.
\end{abstract}

Keywords-Image edge extraction; Cell Neural Network (CNN); style; Digital Image processing;

\section{INTRODUCTION}

Image edge extraction is a very important basis for image analysis area such as image segmentation, the recognition of target area and regional shape extraction plays an important role in engineering application. Cellular neural network (CNN) is a kind of parallel processor, and its application in image model identification will make higher recognition rate in system.

Because there are some defects in the traditional algorithm, $\mathrm{CNN}$ will be applied to the edge detection of gray image. Because $\mathrm{CNN}$ is a kind of neural network parallel processor based on local connection of neurons[1]. The difference with the fully interconnected Hopfield neural network is, each neurons only connects with neighboring neurons and neurons in all of the adjacent connection structure and connection weights are the same. Therefore, in respect of hardware we can design CNN parallel processor by the same circuit element array, the circuit design of the isomorphism of the array is conducive to VLSI implementation.

Edge is one of the important characteristics of image, and it is also the element of several research areas, such as computer vision and pattern identification. In the area of image processing, the $\mathrm{CNN}$ has a broad developing space.

The edge of the image is refer to the gray of pixel level around, color or texture with step changes or changes in the roof of the pixel set, as a result of some physical properties and surface characteristics, the outcome of geometry and reflectance change. It mainly exists between objectives and objectives, objectives and background, region and region, element and element [2]. In the image, the gray value of two pixels is big difference, the "big difference" is that it is relative to the gray value of the surrounding pixels. Edge can be divided into step edge, pulse edge and roof edge [3].
In recent years, extracting the image edge by neural network has become a new branch, Chao proposed detecting the image edge by Hopfield neural network[4], and using cellular neural network to detect the image edge proposed by Berkeley L.O.Chua and L. Yang of the University of California of the United States, in 1988[5]. Like neural network, it is a largescale nonlinear analog circuit which has real-time signal processing ability; Like cellular automata, which consists of a large number of cellular composition and only allows direct communication between adjacent cells. Each cell is only composed of a linear capacitor, a nonlinear voltage control current source and a small amount of resistive linear circuit elements[6]. Application of cellular neural network are different from the traditional methods of image processing, because it firstly map the image into a neural network, each pixel is a neuron, namely the scale are the same as the pending image, if the pending image contains $M^{*} \mathrm{~N}$ pixel, then the cellular neural network also contains $\mathrm{M}^{*} \mathrm{~N}$ neurons. In image processing, the state of each neuron is iterated according to its dynamic equation, until the whole network converges and outputs the two value signal. The basic idea of this method is summarized as follows, the whole image function is transformed into a dynamic system, and the dynamic equation guides the change of the minimum energy direction defined by the neural network to the cellular neural network to realize the edge extraction[7].

\section{DEFINITION AND THEOREM IN CELL NEURAL NETWORK}

Cellular neural network algorithm and the traditional methods are completely different, because the image is firstly mapped into a neural network, each pixel is a neuron, and then guide the state of the neuron to the direction of the lowest energy neural network as defined by neural network through the dynamic equation to achieve edge detection. For cellular neural networks has the characteristics of parallel computing, and is easy to be realized by hardware. so it have greater potential than traditional methods, and is the main research directions of hardware implementation as well. Hopfield is a global neural network, the state of every point are related to the entire image, the CNN network is a locally connected network topology model, it is much simpler than other interconnection model. $\mathrm{CNN}$ is also a positive feedback system, it can ensure good output characteristics.

The basic circuit unit of cell neural network is called cells, which comprises linear and nonlinear circuit element, its typical structure is linear capacitance, linear resistor, linear and nonlinear controller source and independent power supply. 
the network has a continuous time propagation effects [8]. A CNN is a spatial arrangement of local connected cells, each of which has an input and an output and the dynamic state of the dynamic system, as shown in Figure 1.

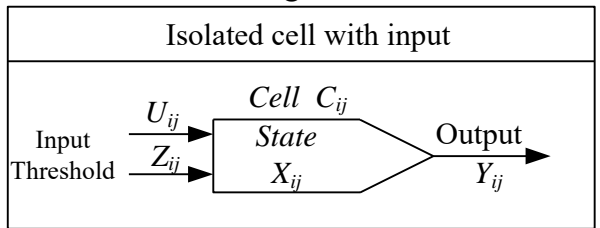

Figure 1. Dynamic system of CNN

Definition 1 The cells in the ith row and the jth column of a two-dimensional $\mathrm{M} \times \mathrm{N}$ cellular neural networks is denoted as $c(i, j)$.

Definition $2 \mathrm{r}$ neighborhood (influence domain) of $c(i, j)$ is defined as

$N_{r}(i, j)=\{C(k, l) \mid \max \{|k-i|,|l-j|\} \leq r, 1 \leq k \leq M, 1 \leq l \leq N\}$

Where $r$ is positive integer. The cellular neural network formula is derived as follows, state equation is

$$
\begin{aligned}
C \frac{d v_{x i j}(t)}{d t}=-\frac{1}{R x} v_{x i j}(t) & +\sum_{c(k, l) \in N_{r}(i, j)} A(i, j ; k, l) v_{y k l}(t) \\
& +\sum_{c(k, l) \in N_{r}(i, j)} B(i, j ; k, l) v_{u k l}(t)+I
\end{aligned}
$$

Output equation is

$$
v_{y i j}(t)=\frac{1}{2}\left(\left|v_{x i j}(t)+1\right|-\left|v_{x i j}(t)-1\right|\right), \quad 1 \leq i \leq M, 1 \leq j \leq N
$$

Input equation is

$$
v_{u i j}=E_{i j} \quad 1 \leq i \leq M, 1 \leq j \leq N
$$

The constraint conditions

$$
\left|v_{u i j}\right| \leq 1, \quad 1 \leq i \leq M, 1 \leq j \leq N
$$

Parameter hypothesis

$$
\begin{aligned}
& A(i, j ; k, l)=A(k, l ; i, j), 1 \leq i, k \leq M, 1 \leq j, l \leq N \\
& R x>0 \quad C>0
\end{aligned}
$$

Each symbol in equation $(1) \sim(5)$ is defined as follows, $V_{x i j}(t), V_{y i t}(t)$ and $V_{u i j}(t)$ are the state of $c(i, j)$, output and input signal (or voltage); I is the bias current (or threshold); $c$ and $\mathrm{Rx}$ are linear capacitor and resistor; $A(i, j, k, l)$ and $B(i, j, k, l)$ are the feedback operator and control operator; $f$ is output function, it is given by sectionalized linear function $f(v)=0.5|v+1|-|v-1|$, Its shape is as shown in Figure 2.

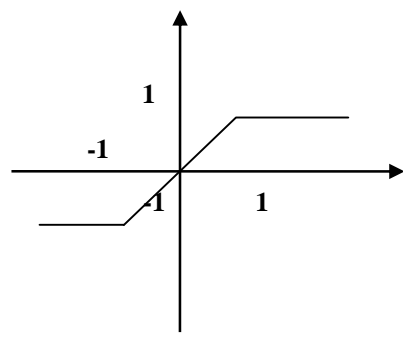

Figure 2. $\mathrm{CNN}$ input function $\mathrm{f}(\mathrm{v})=0.5|\mathrm{v}+1|-|\mathrm{v}-1|$

In order to ensure that the circuit equation $(1) \sim(5)$ hold for all cellular neural network, three very important theorem are verified for reference [5].

Theorem 1 The state of each cell of Vxij in cellular neural networks is bounded for all time $t>0$, the boundary maximum value of the Vmax satisfy condition

$$
V_{\max }=1+R_{x}|I|+R_{x} \max _{1 \leq i \leq M, 1 \leq j \leq N}\left[\sum_{c(k, l) \in N_{r}(i, j)}|A(i, j ; k, l)|+|B(i, j ; k, l)|\right]
$$

Theorem 2 In the transient process, cellular neural network is always tends to a stable equilibrium point. There exists

$$
\lim _{x \rightarrow \infty} v_{x i j}(t)=\text { constant }, \quad 1 \leq \mathrm{i} \leq \mathrm{M}, 1 \leq \mathrm{j} \leq \mathrm{N}
$$

Theorem 3 If the system parameters satisfy $A(i, j ; k$, 1) $>1 / \mathrm{Rx}$, then when the transient process finished, the cellular neural network in each cell will tend to stable equilibrium point, these stable equilibrium point meet the conditions

$$
\begin{array}{r}
\lim _{x \rightarrow \infty}\left|v_{x i j}(t)\right|=\text { constant, } \quad 1 \leq \mathrm{i} \leq \mathrm{M}, 1 \leq \mathrm{j} \leq \mathrm{N} \\
\lim _{x \rightarrow \infty}\left|v_{y i j}(t)\right|= \pm 1, \quad 1 \leq \mathrm{i} \leq \mathrm{M}, 1 \leq \mathrm{j} \leq \mathrm{N}
\end{array}
$$

Theorem 1 and theorem 2 ensures that the cellular neural network circuit will not shock or change into chaos. Theorem 3 ensures the cellular neural networks have two value output, it is necessary to ensure the cellular neural network is successfully applied to image edge detection.

When Applying CNN to image processing, general it need to preprocess on the following equation

A. Convert differential equations into difference equations In equation (1), let $C=R x=1$, then the equivalent differential of equation (1) is

$$
\begin{aligned}
& v_{x i j}(n+1)-v_{x i j}(n)=-v_{x i j}(n)+\sum_{c(k, l) \in N_{r}(i, j)} A(i, j ; k, l) v_{y k l}(n) \\
& \quad+\sum_{c(k, l) \in N_{r}(i, j)} B(i, j ; k, l) v_{u k l}(n)+I
\end{aligned}
$$

Namely

$$
v_{x i j}(n+1)=\sum A(i, j ; k, l) v_{y k l}(n)+\sum B(i, j ; k, l) v_{y k l}(n)+I
$$

B. Pixel range adjustment To guarantee the constraint condition (4) of $\mathrm{CNN}$, transform Gray image pixel value[0, 
$255]$ to the range [-1,1], namely, set $x \in[0,255], s=2 *(x / 255$ $0.5)$, then $s \in[-1,1]$.

\section{DEFINITION AND THEOREM IN CELL NEURAL NETWORK}

By the state equation (1) of the cell, we can see that the dynamic mechanism of CNN include two kinds of dynamic mechanism, output feedback and input control. Because the effect of output feedback depends on the template A, and the effect of the input control is determined by the template B, so the key problem is how to find the appropriate template in $\mathrm{CNN}$ image processing. Some successful templates come from the famous digital image processing algorithms, such as the literature [10]. The method is transfering the low pass filter template directly into cellular neural network parameters; and we use the principle of high pass filter to design network parameters.

Because of high spatial frequency at edge, the high frequency component can be made by high pass filtering method, which can be used to suppress the low frequency component, so that the high frequency component can be enhanced, and the image edge becomes clear, so as to realize the image sharpening. This idea is reflected in the spatial domain processing, it is to make convolution the image and high pass filter template $H$. Equation (12) is a commonly used high pass filter template.

$$
H=\left|\begin{array}{ccc}
-1 & -1 & -1 \\
-1 & 8 & -1 \\
-1 & -1 & -1
\end{array}\right|
$$

Because we should deal with digital images, it is necessary to carry out the cell state equation for differential treatment, so as to better study the dynamic characteristics of the cellular neural network, thus by (10) we have

$$
\begin{aligned}
v_{x i j}(n+1)=v_{x i j}(n) & +\frac{h}{C}\left[\frac{-1}{R_{x}} v_{x i j}(n)+\sum_{c(k, l) \in N_{r}(i, j)} A(i, j ; k, l) v_{y k l}(n)\right. \\
& \left.+\sum_{c(k, l) \in N_{r}(i, j)} B(i, j ; k, l) v_{u k l}(n)+I\right]
\end{aligned}
$$

Where $1 \leq i \leq M, 1 \leq j \leq N$.

The state $v_{x i j}(n)$ of $\mathrm{n}$ time is determined by the initial $v_{x i j}(0)$, The state $v_{x i j}(n)$ of cell $c(i, j)$ at time $\mathrm{n}$ is determined by the initial state $v_{x i j}(0)$, the input $v_{u k l}(c(k, l)) \in N r(i, j)$ and network parameter. When $n \rightarrow \infty$, because the chareacter of cell neural network, the state variable $v_{x i j}$ tend to constant values, and output $y_{x i j}$ tend to +1 or -1 , therefore it can be used to separate the edge point $(+1)$ and non edge (-1) of two states.

In equation(13), $t=n h$, if the step $\mathrm{h}$ is taken too small, it will lead to slow convergence; if it is taken too big, differential equation and differential equation is too large and may even lead to non convergence or convergence to the wrong state. So in the actual treatment process, we can take step $h=1$ and neighborhood range " $3 \times 3$ ", so the simulation will be relatively simple. At the same time, we take the time constant $R \times C$, which reflects the network speed of dynamic process.
Therefore the time constant is 1 ; In the process of compiling algorithm we take $c=1, R x=1$, therefore the time constant is 1 . The initial state of the cell is taken $v_{x i j}(0)=0$. When we take the output vx of cell neural network as the pixel value of image, accordingly, the template $\mathrm{B}$ can be taken as a high pass template $\mathrm{H}$, to get the sharpening effect of image edge. For the constraint condition of CNN is $\left|v_{x i j}\right| \leq 1(1 \leq i \leq M, 1 \leq j \leq N)$, and the gray value of the image is represented by the number between 0 and 255, therefore, Directly to the black (gray value of 0 ) corresponds to $v_{u}=1$, white (gray value is 255 ) corresponding to $v_{u}=-1$. We set black (gray value is 0 ) corresponding to $v_{u}=1$, white (gray value is 255 ) corresponding to $v_{u}=-1$. Without considering the effect of output feedback between inter cell, then formula (13) can be simplified as

$$
v_{x i j}=B * v_{u}+I
$$

Where"*" represent solving convolution. The convolution is that each element of each pixel in the neighborhood multiply with template respectively and sum, The template size is the same as the neighborhood, The matrix, it represent the $3 \times 3$ matrix composed of external input value of neighborhood of the cell, and is written as

$$
v_{u}=\left(\begin{array}{lll}
x_{1} & x_{2} & x_{3} \\
x_{4} & x_{5} & x_{6} \\
x_{7} & x_{8} & x_{9}
\end{array}\right)
$$

Obviously, the value of pixel point $(i, j)$ being investigated is corresponding to $x_{5}$ in $v_{u}$.

(1) when $x_{5}=-1$ or $x_{1}=x_{2}=\cdots=x_{5}=\cdots=x_{9}=1,(i, j)$ is non-edge point, when it is steady state, $v_{x i j} \leq 1, v_{y i j}=-1$.

(2) When $\mathrm{x}_{5}=1$, and any one or more of $x_{1}, x_{2}, x_{3}, x_{4}, x_{6}, x_{7}$, $x_{8}, x_{9}$ is not equal to $1,(i, j)$ is edge point, when it is steady state, $v_{x i j} \leq 1, v_{y i}=-1$.

Through the above two points, then from equation(15) and $B=H$ we can get inequalities, and get the value $I=-0.5$.

In addition, by the property theorem of cellular neural network we can draw a conclusion, when $a(i, j ; k, l)>1 / R x$, when cellular neural networks in steady state it will have two output value, because we have taken $R x=1, a(i, j ; k, l)$ is sure a number greater than 1 , In the process of compiling the program $a(i, j ; k, l)=2$, It has a positive feedback effect. it makes the cell state value corresponding to edge point of image more and more big, after the state $v_{y i j}$ is convergent, its output is 1; It makes the state value of non-edge point of image more and more small, when the state is convergent the output of $v_{y i j}$ is -1 . In order to simplify the design, we take the following set of $\mathrm{CNN}$ parameters. 


$$
\begin{gathered}
\mathrm{A}=\left|\begin{array}{ccc}
0 & 0 & 0 \\
0 & 2 & 0 \\
0 & 0 & 0
\end{array}\right| \mathrm{B}=\left|\begin{array}{ccc}
-1 & -1 & -1 \\
-1 & 8 & -1 \\
-1 & -1 & -1
\end{array}\right| \\
I=0.5
\end{gathered}
$$

Define abbreviations and acronyms the first time they are used in the text, even after they have been defined in the abstract. Abbreviations such as IEEE, SI, MKS, CGS, sc, dc, and rms do not have to be defined. Do not use abbreviations in the title or heads unless they are unavoidable.

\section{REALIZATION OF IMAGE EDGE DETECTION ALGORITHM}

When we have got the network parameters, the compile process of program will process.

First, we will enter a pending two value image, its size is arbitrary. The corresponding parameters of the set template are shown in the formula (16). The initial state is set to 0 . Figure 3 is the flow chart design of the two value image algorithm. Figure 4 is an example of the actual extraction of the image.

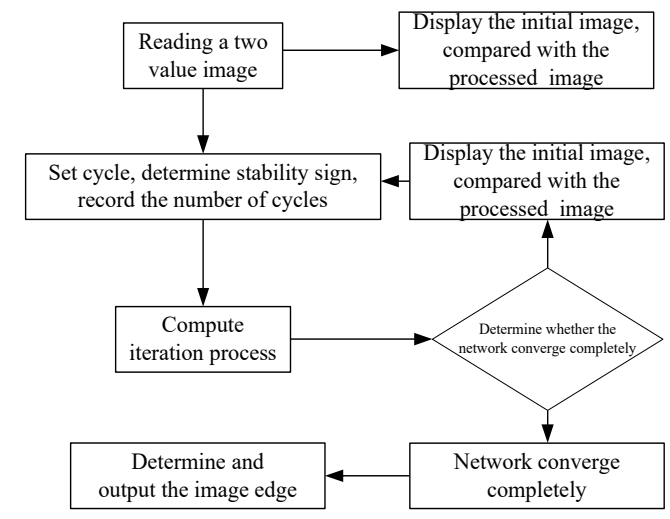

Figure 3. Flow chart of edge extraction of two valued image based on
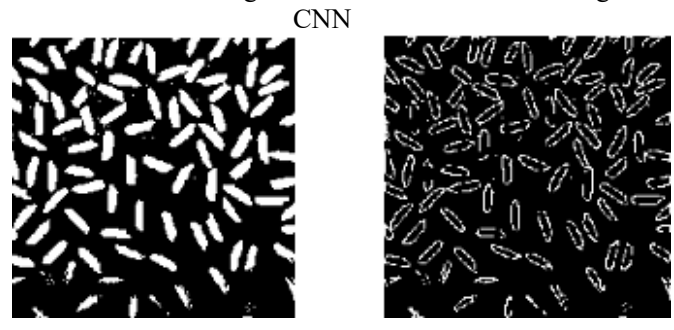

Figure 4. Example of the actual extraction of the image

From figure 4, CNN algorithm has a good effect on image processing with low noise, especially in the extraction of binary image edges. The edges of the images extracted are exquisite.

\section{CONCLUSION}

The theory of traditional algorithm and edge extraction of cellular neural network are analyzed, and then the application of neural network in image processing is studied.CNN algorithm has a good effect on image processing with low noise, especially in the extraction of binary image edges. The edges of the images extracted are exquisite.

Because the cellular neural network is capable of highspeed parallel computing, the processing speed is independent of image size, and it is convenient for hardware implementation. It has great potential to be developed in realtime image processing.

The key of application of $\mathrm{CNN}$ in image processing is the template values, different values have different image processing functions, so the model value is the main problem. The two value image template in $\mathrm{CNN}$ and the edge extraction of gray image are the direction of further research.

$\mathrm{CNN}$ has a lot of advantages in hardware implementation, but if the scale of image is larger, it is difficult to use existing chip to complete the large-scale image synthesis, at present we can only achieve its functional simulation, how to further enhance the performance of chips used, speed up the realization of hardware speed, is also the direction of further research.

\section{ACKNOWLEDGEMENTS}

This work was supported by the follows: The science and technology research project of Hebei (No.QN20171390) and The young foundation of Shijiazhuang Tiedao University. (No. 20153011)

\section{REFERENCES}

[1] L.O.Chua, CNN: .A Vision of Complexity, International J. of Bifurcation and Chaos .1997, pp.77-89

[2] J, M, Liu. Image segmentation based on CNN and its application in medical image. Master's degree thesis of Beijing Normal University. 2000

[3] Y.F.Chen. Retinal vessel image edge extraction based on Wavelet Transform. Master's degree thesis of South China University of Technology. 2016, pp.360-380

[4] Chao C H, Dhawan A P. Edge detection using a Hopfield neural network. Optical Engineering. 1994

[5] Chua LO, Yang L. Cellular neural networks Theory [J], IEEE Trans. Circuits Syst. 1988,

[6] L,C,Jiao. Application and Realization of neural network. Xi'an Electronic and Science University press,2017, pp.111-120

[7] J,F,Ma. Research of image edge detection based on cellular neural network. Science technology and Engineering.2009, pp.15-26 